\title{
Sitafloxacin Expresses Potent Anti-Mycobacterium abscessus Activity
}

\author{
Siyuan $\mathrm{He}^{1,2+}$, Qi Guo ${ }^{1,2+}$, Lan Zhao' ${ }^{1+}$, Liyun $X u^{1}$, Junsheng Fan ${ }^{1,2}$, Wenye $W u^{1}$, \\ Zhemin Zhang ${ }^{1}$, Bing $\mathrm{Li}^{1 *}$ and Haiqing $\mathrm{Chu}^{1,3 *}$
}

${ }^{1}$ Department of Respiratory and Critical Care Medicine, Shanghai Pulmonary Hospital, School of Medicine, Tongji University, Shanghai, China, ${ }^{2}$ School of Medicine, Tongji University, Shanghai, China, ${ }^{3}$ Shanghai Key Laboratory of Tuberculosis,

Shanghai Pulmonary Hospital, School of Medicine, Tongji University, Shanghai, China

\section{OPEN ACCESS}

Edited by:

Hemda Garelick,

Middlesex University, United Kingdom

Reviewed by:

Mehmet Demirci,

KIrklareli University, Turkey

Dinah Binte Aziz,

National University of Singapore,

Singapore

*Correspondence:

Bing Li

libing044162@163.com

Haiqing Chu

chu_haiqing@126.com

tThese authors have contributed equally to this work and share first

authorship

Specialty section:

This article was submitted to

Antimicrobials, Resistance

and Chemotherapy,

a section of the journal

Frontiers in Microbiology

Received: 19 September 2021 Accepted: 14 December 2021

Published: 06 January 2022

Citation:

He S, Guo Q, Zhao L, Xu L, Fan J,

Wu W, Zhang Z, Li B and Chu H (2022) Sitafloxacin Expresses Potent

Anti-Mycobacterium abscessus Activity. Front. Microbiol. 12:779531.

do: $10.3389 /$ fmicb.2021.779531
Therapeutic options for treating Mycobacterium abscessus infections are extremely limited; quinolones are important. The in vitro anti-M. abscessus activities of nine quinolones, emphasizing sitafloxacin, were investigated. Antimicrobial susceptibility testing was performed on 10 non-tuberculous mycobacterium reference strains and 194 clinical, M. abscessus isolates. The activity of sitafloxacin against intracellular M. abscessus residing within macrophages was also evaluated. A checkerboard assay was conducted to determine synergy between sitafloxacin and 10 clinically important antibiotics. Among the nine quinolones tested, sitafloxacin exhibited the greatest antiM. abscessus activity with $\mathrm{MIC}_{50}$ and $\mathrm{MIC}_{90}$ of 1 and $2 \mathrm{mg} / \mathrm{L}$, respectively. Sitafloxacin exerted a bacteriostatic effect on $M$. abscessus and inhibited the intracellular growth of M. abscessus at concentrations equivalent to clarithromycin. No antagonism between sitafloxacin and 10 clinically important anti-M. abscessus antibiotics was evident. In summary, sitafloxacin exhibited a significant advantage relative to other quinolones in inhibiting the growth of $M$. abscessus in vitro, suggesting the potential inclusion of sitafloxacin in new strategies to treat $M$. abscessus infections.

Keywords: Mycobacterium abscessus, in vitro, intracellular, quinolone, sitafloxacin

\section{INTRODUCTION}

The number of infections caused by non-tuberculous mycobacteria (NTM) is increasing globally (Cowman et al., 2019; Johansen et al., 2020; Mourad et al., 2021). Mycobacterium abscessus is an important pathogenic NTM for patients with bronchiectasis, chronic obstructive pulmonary disease and cystic fibrosis. M. abscessus infections are the most challenging to treat due to an intrinsic resistance to many common antibiotics (Fletcher et al., 2016; Degiacomi et al., 2019). No evidence-based antibiotic regimen has been established to date; the cure rate and rate of recurrence are far from satisfactory (Stout et al., 2016; Haworth et al., 2017; Daley et al., 2020). Consequently, there is an urgent need for new therapeutic options.

Sitafloxacin is an oral quinolone, which exhibits excellent antibacterial activity by simultaneously inhibiting DNA gyrase and topoisomerase IV in a broad range of bacteria including mycobacteria (Tomioka et al., 1999; Sato et al., 2003; Amano et al., 2016; Nakajima et al., 2016; Asakura et al., 2019; Kamada et al., 2021). Reports of the successful treatment of cases of M. abscessus-associated pneumonia with drug combinations that included sitafloxacin have provoked a recent interest 
in using sitafloxacin for treating M. abscessus infections (Oka et al., 2021; Takano et al., 2021). Information concerning the anti-M. abscessus activity of sitafloxacin is limited, however.

A systematic evaluation of the antimicrobial activity of sitafloxacin against a large number of clinical, M. abscessus isolates was undertaken for the first time in the present study. Sitafloxacin was active against $M$. abscessus growing in vitro; sitafloxacin exhibited the lowest minimum inhibitory concentration (MIC) among nine quinolones tested. Importantly, sitafloxacin was especially effective in inhibiting the growth of organisms growing intracellularly, i.e., in macrophages. Sitafloxacin did not antagonize the activity of antibiotics most frequently used to treat $M$. abscessus infections. These findings demonstrate the significant advantages of sitafloxacin over other quinolones, which may provide a new approach to treating M. abscessus infections.

\section{MATERIALS AND METHODS}

\section{Bacterial Strains}

A total of 10 NTM reference strains and 194 clinical, M. abscessus isolates were evaluated. The following reference strains: M. abscessus subsp. abscessus (ATCC 19977), M. avium (ATCC 25291), M. intracellulare (ATCC 13950), M. kansasii (ATCC 12478), M. fortuitum (ATCC 6841), M. gordonae (ATCC 14470), M. scrofulaceum (ATCC19981), M. peregrinum (ATCC700686), and M. xenopi (ATCC19250) were purchased from the American Type Culture Collection (ATCC; VA, United States). M. abscessus subsp. massiliense (CIP108297) was purchased from the Biological Resource Center of Institut Pasteur (CIP; Paris, France). Detailed information regarding the clinical isolates was provided in a previous study (Guo et al., 2020). All strains were grown at $37^{\circ} \mathrm{C}$ on Middlebrook $7 \mathrm{H} 10$ agar plates supplemented with $10 \%$ OADC and $0.2 \%$ glycerol, or with continuous shaking in Middlebrook 7H9 broth supplemented with 10\% OADC and $0.05 \%$ Tween 80 . Middlebrook 7H9 broth, M7H10 agar, cationadjusted Mueller-Hinton II broth and OADC were purchased from Becton Dickinson and Company (NJ, United States).

\section{Antimicrobial Agents}

Clarithromycin, azithromycin, amikacin, cefoxitin, imipenem, tigecycline, linezolid, and ciprofloxacin were purchased from Sigma-Aldrich Company (St. Louis, MO, United States). Bedaquiline was purchased from Biopharmaleader (Biopharmaleader, China). Nemonoxacin was purchased from Zhejiang Pharmaceutical Co., Ltd. (Xinchang Pharmaceutical Factory, Zhejiang, China). All remaining antibiotics were purchased from MCE (MedChemExpress, Monmouth Junction, NJ, United States). Sitafloxacin, clarithromycin, clofazimine, bedaquiline, rifabutin and sparfloxacin were solubilized in $100 \%$ DMSO; levofloxacin and nemonoxacin were dissolved in $1 \% \mathrm{NAOH}$; azithromycin was dissolved in absolute ethanol; and the remaining antibiotics were prepared in de-ionized water. The antibiotics were aliquoted, stored at $-20^{\circ} \mathrm{C}$ and serially diluted just prior to experimental use.

\section{Minimum Inhibitory Concentration and Minimum Bactericidal Concentration Determination}

Antibiotic susceptibility was determined by the broth microdilution method according to Clinical and Laboratory Standards Institute (CLSI) document M24-A2 (Woods et al., 2011). Briefly, individual M. abscessus colonies were picked from M7H10 agar plates, grown to logarithmic phase in M7H9 broth, diluted to McFarland 0.5 with sterile saline, and adjusted to $1 \times 10^{5}$ to $5 \times 10^{5} \mathrm{CFU} / \mathrm{ml}$ in cationadjusted Mueller-Hinton broth (CAMHB). Nine quinolones (ciprofloxacin, levofloxacin, moxifloxacin, nemonoxacin, sitafloxacin, gatifloxacin, delafloxacin, garenoxacin, and sparfloxacin) were tested. Antibiotics were serially diluted 1:2 in a 96-well microtiter plate (working concentrations ranged from 0.25 to $64 \mathrm{mg} / \mathrm{L}$ ), and $100 \mu \mathrm{l}$ of bacteria suspended in CAMHB was added to each well. The microtiter plates were sealed with parafilm and rapid growing mycobacteria were incubated for $3-5$ days at $37^{\circ} \mathrm{C}$ in ambient air. Slow growing mycobacteria were incubated for 7-10 days, or the time it took for the control wells without antibiotics to exhibit visible growth. MIC was defined as the minimum drug concentration at which no visual bacterial growth occurred (Supplementary Figure 1).

M. abscessus subsp. abscessus ATCC 19977 and subsp. massiliense CIP 108297 were used in assays to determine the MBC of the following seven quinolones: sitafloxacin, ciprofloxacin, levofloxacin, moxifloxacin, nemonoxacin, gatifloxacin, and sparfloxacin. Delafloxacin and garenoxacin were not included in this analysis due to their high MIC values. The contents of each microtiter plate well containing a drug concentration greater than the MIC were evenly suspended on day 4 following completion of the antimicrobial susceptibility test. One hundred microliter aliquots of each well were cultured on Middlebrook 7 H10 supplemented with $0.2 \%$ glycerol and OADC enrichment. CFUs were quantified after an additional 5 days incubation at $37^{\circ} \mathrm{C}$. The $\mathrm{MBC}$ values were defined as the minimum drug concentration that prevented $99.9 \%$ bacterial growth expressed in $\mathrm{CFU} / \mathrm{ml}$. An antibiotic was considered bactericidal if the MBC/MIC ratio was $\leq 4$, or bacteriostatic if the ratio was $>4$.

\section{Anti-mycobacterial Drug Synergy}

Synergy between sitafloxacin and clarithromycin, azithromycin, amikacin, linezolid, clofazimine, imipenem, tigecycline, bedaquiline, cefoxitin, and rifabutin was assessed in vitro using the broth microdilution chequerboard titration technique as previously described (Kaushik et al., 2017). M. abscessus subsp. abscessus reference strain ATCC 19977, M. abscessus subsp. massiliense reference strain CIP 108297, and six clinical isolates (three subsp. abscessus and three subsp. massiliense) were used for evaluation. Synergy test results were interpreted based upon the fractional inhibitory concentration index (FICI), calculated using the following formula: FICI $=($ MIC of antibiotic A in combination/MIC of antibiotic A alone) + (MIC of sitafloxacin in the combination/MIC of sitafloxacin alone). Drug interactions were classified as: 
synergy (FICI $\leq 0.5)$, indifference $(0.5<$ FICI $\leq 4)$ and antagonism (FICI $>4.0)$.

\section{Intracellular Killing Assay}

Mouse peritoneal macrophages, frequently used to examine the factors that affect the intracellular replication of mycobacterium species, were obtained by methods previous described by us (Zhang et al., 2020). The cells were infected with M. abscessus (multiplicity of infection $=5$ ) suspended in RPMI 1,640 medium supplemented with $10 \%$ fetal bovine serum (FBS). After $4 \mathrm{~h}$ incubation at $37^{\circ} \mathrm{C}$ in $5 \% \mathrm{CO} 2$, the cells were washed three times with warm phosphate-buffered saline to remove the extracellular organisms. Fresh RPMI 1,640 medium with 10\% FBS and sitafloxacin, moxifloxacin and clarithromycin at $0.2,1,5$, and
$10 \mu \mathrm{g} / \mathrm{ml}$ was then added; infected control cells were treated with medium and 10\% FBS alone. The cells were lysed with $0.05 \%$ sodium dodecyl sulfate at 4,24 , or $48 \mathrm{~h}$ postinfection and the CFUs were quantified by plating serial dilutions of lysates on $7 \mathrm{H} 10$ agar plates. Cell viability was evaluated by trypan blue exclusion before and after infection or drug treatment at each time point.

\section{Statistical Analysis}

Statistical differences between study groups were determined with Mann-Whitney $U$ - test and Student's non-parametric test $P<0.05$ was considered significant. Computations were performed using GraphPad Prism 8 (GraphPad Software, San Diego, CA).

TABLE 1 | MIC of 9 quinolones for 194 clinical M. abscessus isolates.

\begin{tabular}{|c|c|c|c|c|c|c|c|c|c|c|c|c|c|}
\hline Antibiotics & MIC range (mg/L) & \multicolumn{10}{|c|}{ Number of isolates exhibiting the MIC (mg/L) of quinolones indicated } & $\mathrm{MIC}_{50}(\mathrm{mg} / \mathrm{L})^{\mathrm{a}}$ & $\mathrm{MIC}_{90}(\mathrm{mg} / \mathrm{L})^{\mathrm{a}}$ \\
\hline Ciprofloxacin & $1->64$ & 0 & 0 & 1 & 8 & 58 & 69 & 29 & 12 & 14 & 3 & 8 & 32 \\
\hline Levofloxacin & $2->64$ & 0 & 0 & 0 & 1 & 6 & 39 & 62 & 50 & 28 & 8 & 16 & 64 \\
\hline Moxifloxacin & $0.5-16$ & 0 & 1 & 10 & 41 & 76 & 49 & 14 & 3 & 0 & 0 & 4 & 8 \\
\hline Delafloxacin & $32->64$ & 0 & 0 & 0 & 0 & 0 & 0 & 0 & 3 & 23 & 168 & $>64$ & $>64$ \\
\hline Garenoxacin & $32->64$ & 0 & 0 & 0 & 0 & 0 & 0 & 0 & 1 & 18 & 168 & $>64$ & $>64$ \\
\hline Sparfloxacin & $1->64$ & 0 & 0 & 2 & 6 & 10 & 28 & 70 & 44 & 26 & 8 & 16 & 64 \\
\hline
\end{tabular}

${ }^{{ }^{a}} \mathrm{MIC}_{50}$ and MIC 90 : the concentrations at which 50 and $90 \%$ of the clinical isolates tested, respectively, were inhibited.

TABLE 2 | MIC distribution of sitafloxacin for 194 clinical, M. abscessus isolates.

\begin{tabular}{|c|c|c|c|c|c|c|c|c|c|}
\hline \multirow[t]{2}{*}{ M. abscessus } & \multirow{2}{*}{$\begin{array}{l}\text { No. of } \\
\text { isolates }\end{array}$} & \multicolumn{6}{|c|}{ Number of isolates exhibiting the MIC (mg/L) of sitafloxacin indicated } & \multirow{2}{*}{$\begin{array}{c}\mathrm{MIC}_{50} \\
(\mathrm{mg} / \mathrm{L})^{\mathrm{a}}\end{array}$} & \multirow{2}{*}{$\begin{array}{l}\mathrm{MIC}_{90} \\
(\mathrm{mg} / \mathrm{L})^{2}\end{array}$} \\
\hline & & 0.25 & 0.5 & 1 & 2 & 4 & 8 & & \\
\hline subsp. abscessus ${ }^{b}$ & 148 & 11 & 55 & 63 & 15 & 3 & 1 & 1 & 2 \\
\hline subsp. Massiliense & 46 & 1 & 10 & 18 & 16 & 1 & 0 & 1 & 2 \\
\hline
\end{tabular}

${ }^{a}{ }^{M} I_{50}$ and $M I_{90}$, the sitafloxacin concentration at which 50 and $90 \%$ of the clinical isolates tested, respectively, were inhibited. ${ }^{b} \mathrm{M}$. abscessus subsp. abscessus was significantly more sensitive than subsp. massiliense to sitafloxacin treatment, $P<0.001$.

TABLE 3 | MIC of 9 quinolones for NTM reference strains.

\begin{tabular}{|c|c|c|c|c|c|c|c|c|c|}
\hline Species & STFXa & CIP & LFX & MFX & NOX & GXT & DLX & GAX & SPX \\
\hline M. massiliense & 1 & 8 & 16 & 4 & 4 & 8 & $>64$ & $>64$ & 32 \\
\hline M. avium & 0.5 & 8 & 8 & 1 & 8 & 4 & 4 & 8 & 1 \\
\hline M. kansasii & 1 & 4 & 0.5 & 0.125 & 64 & 0.5 & 4 & 2 & 0.25 \\
\hline M. szulgai & 1 & 2 & 1 & 0.06 & 16 & 0.5 & 4 & 2 & 0.25 \\
\hline M. fortuitum & 0.06 & 0.25 & 0.25 & 0.06 & 0.5 & 0.125 & 1 & 0.5 & 0.125 \\
\hline M. peregrinum & $<0.06$ & 0.125 & 0.25 & $<0.06$ & 0.25 & 0.125 & 0.5 & 0.25 & 0.125 \\
\hline M. xenopi & $<0.06$ & $<0.06$ & 0.125 & $<0.06$ & $<0.06$ & $<0.06$ & 0.25 & 0.25 & $<0.06$ \\
\hline
\end{tabular}

a STFX, sitafloxacin; CIP, ciprofloxacin; LFX, levofloxacin; MFX, moxifloxacin; NOX, nemonoxacin; GXT, gatifloxacin; DLX, delafloxacin; GAX, garenoxacin; SPX, sparfloxacin. 


\section{RESULTS}

\section{Sitafloxacin Expresses Superior Anti-M. abscessus Activity}

A total of 194 clinical, M. abscessus isolates (148 subsp. abscessus and 46 subsp. massiliense) were collected. Compared to eight other quinolones, sitafloxacin exhibited the greatest in vitro activity; the MIC ranged from 0.25 to $4 \mathrm{mg} / \mathrm{L}$, the $\mathrm{MIC}_{50}$ and MIC $_{90}$ were 1 and $2 \mathrm{mg} / \mathrm{L}$, respectively (Table 1). Subspecies analysis found that $M$. abscessus subsp. abscessus isolates were significantly more sensitive to sitafloxacin than $M$. abscessus subsp. massiliense isolates (Table 2). The minimum bactericidal concentration (MBC)/MIC ratio evidenced the bacteriostatic activity expressed by sitafloxacin toward $M$. abscessus, which is the same as that exhibited by other quinolones (Supplementary Table 1). Sitafloxacin also exhibited high activity toward rapidly growing (i.e., M. fortuitum and $M$. peregrinum), as well as slowly growing (i.e., M. intracellulare, M. avium, M. kansasii, M. szulgai, $M$. xenopi, and $M$. scrofulaceum), NTM reference strains (Table 3).

\section{Sitafloxacin Inhibits Intracellular \\ M. abscessus Growth}

The effects of sitafloxacin, moxifloxacin and clarithromycin on the survival of $M$. abscessus subsp. abscessus ATCC 19977 and subsp. massiliense CIP 108297 residing in primary murine peritoneal macrophages were evaluated (Figures 1, 2). Sitafloxacin, moxifloxacin, and clarithromycin inhibited the intracellular growth of $M$. abscessus for both subspecies tested; inhibition was dose-dependent. Sitafloxacin appeared to exert a greater effect than moxifloxacin at most concentrations, albeit no statistical difference was observed. The effect of sitafloxacin on the intracellular growth of $M$. abscessus subspecies was comparable to that found for clarithromycin.

\section{The Indifferent Interaction Between Sitafloxacin and 10 Clinically Important Anti-M. abscessus Antibiotics}

Synergy between sitafloxacin and 10 antibiotics frequently used clinically to treat $M$. abscessus infections was tested using two reference strains and six clinical isolates. Sitafloxacin did not antagonize any of the other antibiotics tested; all interactions were indifferent [fractional inhibitory concentration index $($ FICI $)=0.75-2]$ (Table 4).

\section{DISCUSSION}

The antibacterial activity of quinolones (e.g., moxifloxacin and levofloxacin) commonly used to treat $M$. abscessus infections is unsatisfactory (Choi et al., 2012a). Sitafloxacin, a new fluoroquinolone that is effective against a broad range of bacteria, has the advantages of oral administration
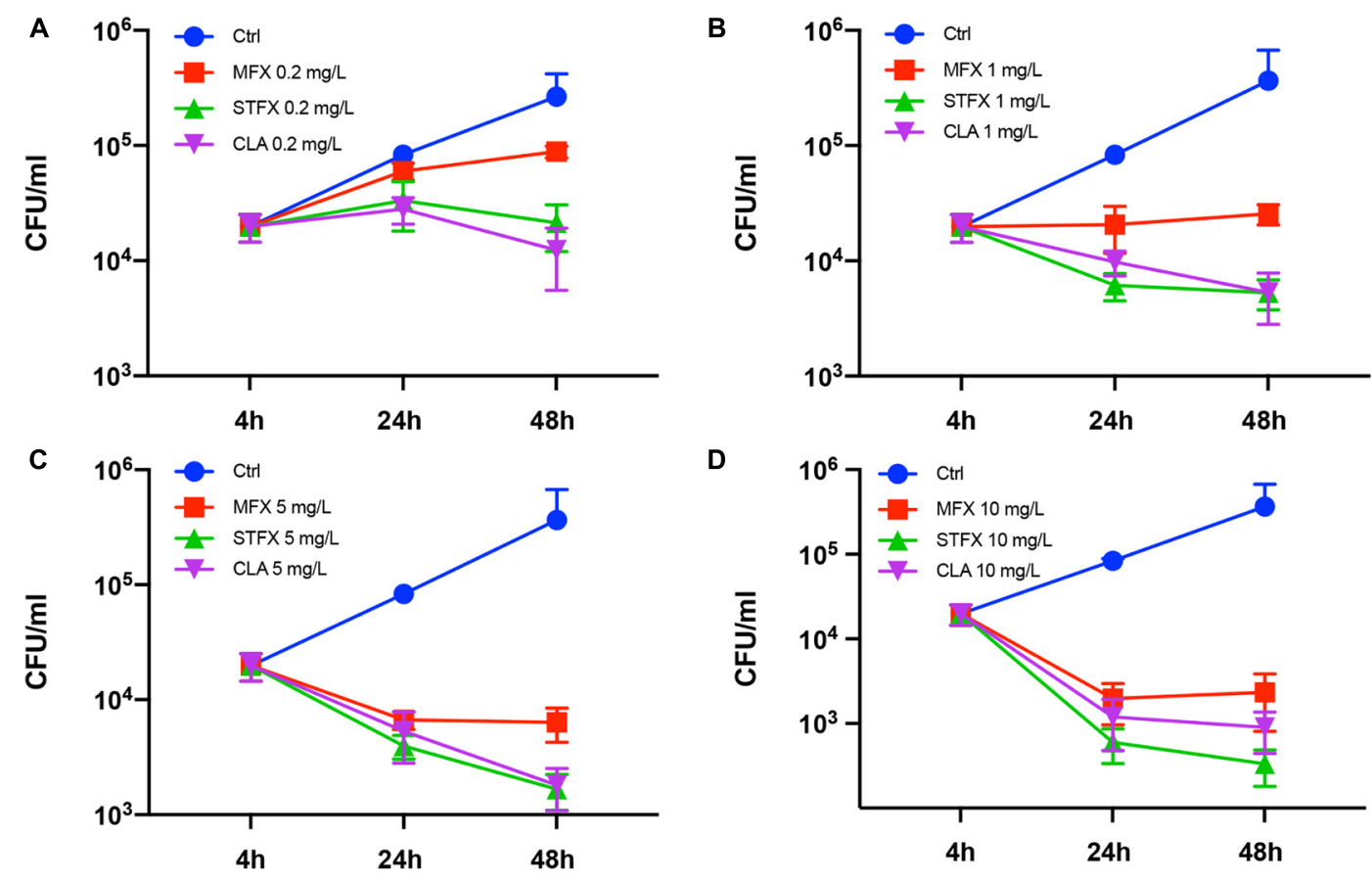

M. abscessus subsp. abscessus ATCC19977

FIGURE 1 | Antimicrobial activities of sitafloxacin, moxifloxacin and clarithromycin against M. abscessus subsp. abscessus ATCC19977 growing intracellularly. Antibiotics at 0.2 (A), 1 (B), 5 (C), and10 (D) mg/L final concentrations were added to cultures of $M$. abscessus-infected macrophages. CFUs were quantified after 4, 24, and 48 h incubation. Ctrl, control; STFX, sitafloxacin; MFX, moxifloxacin; CLA, clarithromycin. 
and superior safety (Wu et al., 2014; Amano et al., 2016; Nakajima et al., 2016; Miyazaki et al., 2019; Mori et al., 2020; Li et al., 2021a,b). Importantly, sitafloxacin exhibits antibacterial activity against $M$. tuberculosis and $M$. avium both in vitro and in vivo (Tomioka et al., 1999; Sato et al., 2003; Asakura et al., 2019). Cases of M. abscessus infection successfully treated with a drug combination that included sitafloxacin were reported (Oka et al., 2021; Takano et al., 2021). The efficacy of sitafloxacin alone in treating $M$. abscessus infections, however, remains to be evaluated.
Antibiotic susceptibility testing of 9 quinolones, including sitafloxacin, was conducted on 194 clinical, M. abscessus isolates in the present study. Sitafloxacin exhibited the lowest MIC ranging from 0.25 to $4 \mathrm{mg} / \mathrm{L}$, and $\mathrm{MIC}_{50}$ and $\mathrm{MIC}_{90}$ of 1 and $2 \mathrm{mg} / \mathrm{L}$, respectively. Kamada K. and coworkers reported similar results: the MIC of sitafloxacin for $M$. abscessus ranged from 0.25 to $>4 \mathrm{mg} / \mathrm{L}$, with $\mathrm{MIC}_{50}=1 \mathrm{mg} / \mathrm{L}$ and $\mathrm{MIC}_{90}=4 \mathrm{mg} / \mathrm{L}$ (Kamada et al., 2021). However, other investigators determined the $\mathrm{MIC}_{50}$ and $\mathrm{MIC}_{90}$ of sitafloxacin against $M$. abscessus to be 3.13 and $6.25 \mathrm{mg} / \mathrm{L}$, respectively, though their sample size was small (Saito et al., 1994).
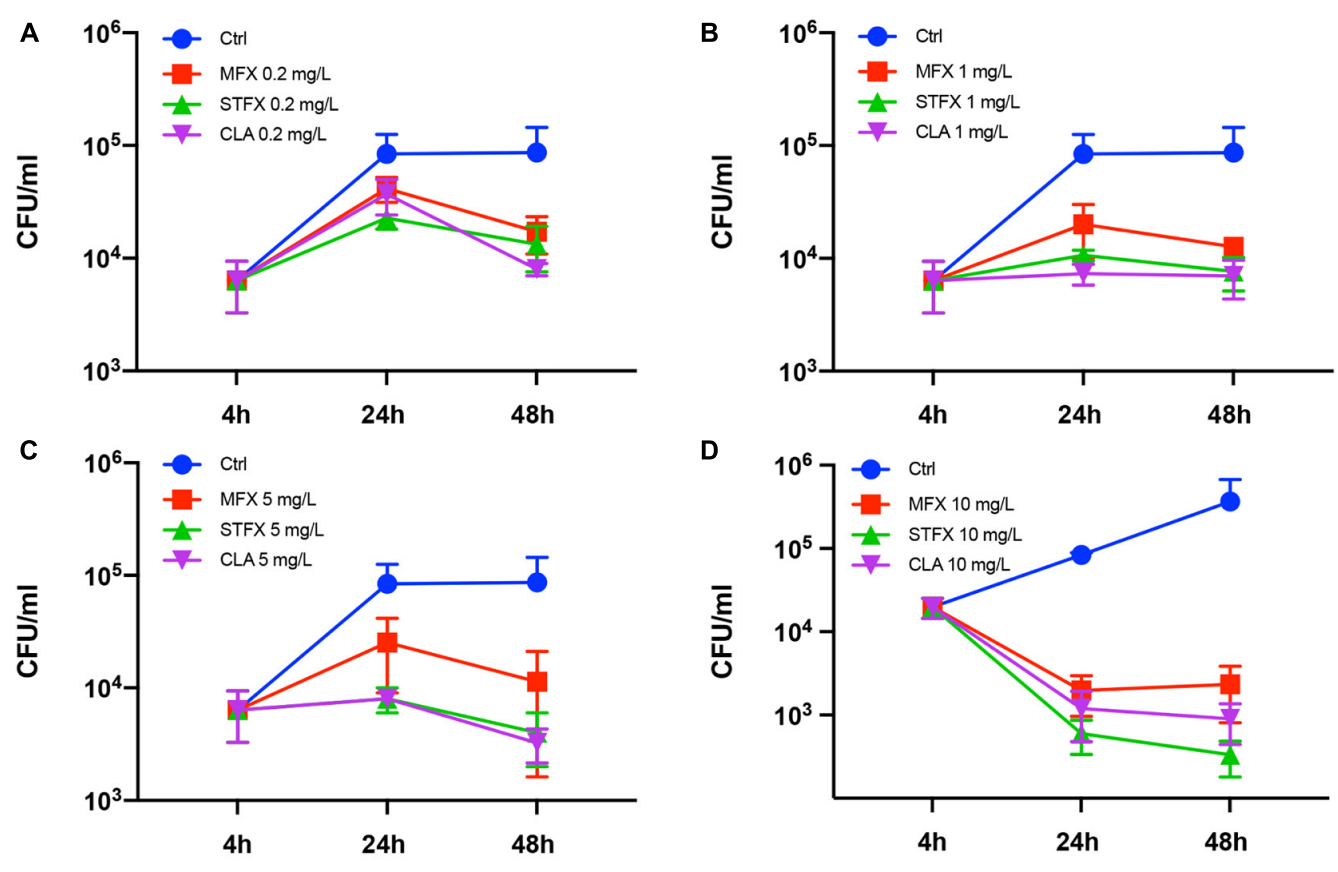

M. abscessus subsp. massiliense CIP108297

FIGURE 2 | Antimicrobial activities of sitafloxacin, moxifloxacin and clarithromycin against intracellular M. abscessus subsp. massiliense CIP 108297. Antibiotics at 0.2 (A), 1 (B), 5 (C), and10 (D) mg/L final concentrations were added to cultures of $M$. abscessus-infected macrophages. Bacterial CFUs were quantified after drug-treated and control M. abscessus-infected cells were cultured for 4, 24, and 48 h. Ctrl, control; STFX, sitafloxacin; MFX, moxifloxacin; CLA, clarithromycin.

TABLE 4 | Interaction of sitafloxacin with antibiotics frequently used to treat clinical M. abscessus infections.

\begin{tabular}{|c|c|c|c|c|c|c|c|c|c|c|c|}
\hline \multirow[t]{2}{*}{ Isolate } & \multirow[t]{2}{*}{ Subspecies } & \multicolumn{10}{|c|}{$\mathrm{FICl}^{\mathrm{a}}$} \\
\hline & & $\begin{array}{c}\text { STFX } \\
+ \text { CLA }^{b}\end{array}$ & $\begin{array}{c}\text { STFX } \\
+ \text { AZM }\end{array}$ & $\begin{array}{l}\text { STFX } \\
+ \text { AMK }\end{array}$ & $\begin{array}{l}\text { STFX } \\
+ \text { LZD }\end{array}$ & $\begin{array}{l}\text { STFX } \\
+ \text { CFZ }\end{array}$ & $\begin{array}{l}\text { STFX } \\
+ \text { IPM }\end{array}$ & $\begin{array}{l}\text { STFX } \\
+ \text { TGC }\end{array}$ & $\begin{array}{c}\text { STFX } \\
+ \text { BDQ }\end{array}$ & $\begin{array}{l}\text { STFX } \\
+ \text { FOX }\end{array}$ & $\begin{array}{l}\text { STFX } \\
+ \text { RFB }\end{array}$ \\
\hline 156 & abscessus & 1 & 0.75 & 1 & 1 & 1.5 & 2 & 2 & 2 & 1.5 & 2 \\
\hline 204 & abscessus & 0.75 & 1 & 1.5 & 1 & 2 & 1.5 & 2 & 2 & 2 & 1.5 \\
\hline 206 & abscessus & 1 & 1 & 1.5 & 1.5 & 2 & 1.5 & 1.5 & 1 & 1.5 & 1.25 \\
\hline 98 & massiliense & 0.75 & 1 & 1 & 1.5 & 1.5 & 1 & 1.5 & 2 & 2 & 1.5 \\
\hline 163 & massiliense & 1 & 0.75 & 2 & 1.5 & 2 & 2 & 1.5 & 2 & 2 & 1 \\
\hline 220 & massiliense & 1 & 1 & 1 & 1.5 & 2 & 1.5 & 1.5 & 1.5 & 2 & 1.5 \\
\hline ATCC 19977 & abscessus & 0.75 & 1 & 1 & 1 & 1.5 & 1 & 1.5 & 2 & 1.25 & 1 \\
\hline CIP 108297 & massiliense & 0.75 & 0.75 & 1.25 & 1.5 & 1 & 1 & 1.5 & 2 & 1.5 & 1 \\
\hline
\end{tabular}

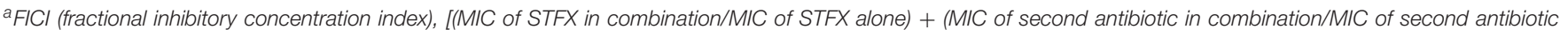

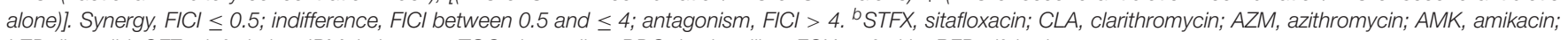
LZD, linezolid; CFZ, clofazimine; IPM, imipenem; TGC, tigecycline; BDQ, bedaquiline; FOX, cefoxitin; RFB, rifabutin. 
A phase I clinical trial investigating the pharmacokinetics and tolerance to sitafloxacin found that a serum concentration of $1 \mathrm{mg} / \mathrm{L}$ was safely attained and well tolerated by healthy male volunteers administered a single $100 \mathrm{mg}$ dose orally ( $\mathrm{Li}$ et al., 2021b). This serum concentration is similar to the $\mathrm{MIC}_{50}$ of sitafloxacin for $M$. abscessus found in the current study, thus a safe and effective dose can be achieved for treating M. abscessus clinically. The antibacterial activity of sitafloxacin against other NTM reported here was superior or equal to that of other quinolones, which is consistent with the results of previous studies of other investigators (Saito et al., 1994; Tomioka et al., 1999; Asakura et al., 2019; Kamada et al., 2021). Notably, sitafloxacin exhibited an average MIC of $1.24 \mathrm{mg} / \mathrm{L}$ for isolates that were resistant to moxifloxacin ( $\mathrm{MIC} \geq 4 \mathrm{mg} / \mathrm{L}$ ) in the study presented here (Supplementary Table 2). These results highlight the efficacy of sitafloxacin in treating NTM infections.

M. abscessus is phagocytized and replicates within macrophages thus circumventing host defenses during infection. The ability of an antibiotic to kill intracellular M. abscessus is essential for treatment. Moxifloxacin (the most effective, available quinolone) and clarithromycin (the current cornerstone drug for $M$. abscessus treatment) were selected and compared to sitafloxacin in an intracellular bactericidal assay. Sitafloxacin exhibited intracellular anti-M. abscessus (subsp. abscessus and subsp. massiliense) activity that was comparable to clarithromycin, and which tended to be greater than moxifloxacin albeit without reaching statistical significance. Notably, five severe $M$. abscessus cases recently treated with a combination of clarithromycin and sitafloxacin achieved good clinical and microbiological outcomes (Takano et al., 2021).

Multidrug combinations are required for treatment of M. abscessus infections (Alangaden and Lerner, 1997; Haworth et al., 2017; Daley et al., 2020). Sitafloxacin is an attractive therapeutic option though optimal effectiveness may necessitate its incorporation into a multidrug treatment plan. In the current study, no antagonism was observed between sitafloxacin and 10 clinically important antibiotics that are often used to treat M. abscessus infections. This suggests that sitafloxacin could easily be integrated into current anti-M. abscessus drug combinations. A greater number of sitafloxacin susceptible M. abscessus subsp. abscessus than M. abscessus subsp. massiliense isolates is of particular interest since infections caused by the former are more difficult to treat and exhibit a worse prognosis due to the induction of clarithromycin resistance (Koh et al., 2011; Choi et al., 2012b). Additional studies addressing this issue are envisioned.

All clinical isolates used in this study were obtained from a single sentinel hospital that receives NTM cases from throughout China. The derivation of all isolates from this single source and

\section{REFERENCES}

Alangaden, G. J., and Lerner, S. A. (1997). The clinical use
of fluoroquinolones for the treatment of mycobacterial
diseases. Clin. Infect. Dis. 25, 1213-1221. doi: 10.1086/
516116

a potential lack of genetic diversity represent shortcomings of this study. Nonetheless, sitafloxacin expresses a high level of antiM. abscessus activity in vitro. Moreover, sitafloxacin is compatible with other antibiotics most frequently used to treat $M$. abscessus infections. As such, sitafloxacin is a potential candidate to include in novel therapeutic anti-M. abscessus regimens.

\section{DATA AVAILABILITY STATEMENT}

The original contributions presented in the study are included in the article/Supplementary Material, further inquiries can be directed to the corresponding author/s.

\section{AUTHOR CONTRIBUTIONS}

HC, BL, SH, QG, LZ, and ZZ conceived and designed the work. LX, JF, and QG collected the bacterial isolates and compiled the data. $\mathrm{SH}$ and $\mathrm{QG}$ performed the experiments. WW, BL, and JF analyzed and interpreted the data. QG and WW were responsible for preparing the figures in the manuscript. SH, QG, and $\mathrm{BL}$ wrote the manuscript, which was reviewed, edited, and approved by all authors.

\section{FUNDING}

This work was funded by grants provided by the: National Natural Science Foundation of China (Nos. 81672063, 81971973, and 81800003), the Natural Science Foundation of Shanghai (Nos. 19ZR1442800, 20ZR1447200, and 21ZR1453600), the Science and Technology Innovation Project of Shanghai (Nos.18411970600, 19411969600, 20Y11903200, and 21Y11900700), the Development Fund for Shanghai Talents (No. 2019112), and the General Project of Shanghai Municipal Health Commission (No. 201940229).

\section{ACKNOWLEDGMENTS}

We sincerely thank Stephen H. Gregory (Providence, Rhodes Island, United States) for his help editing this manuscript.

\section{SUPPLEMENTARY MATERIAL}

The Supplementary Material for this article can be found online at: https://www.frontiersin.org/articles/10.3389/fmicb. 2021.779531/full\#supplementary-material

Amano, A., Kishi, N., Koyama, H., Matsuzaki, K., Matsumoto, S., Uchino, K., et al (2016). In vitro activity of sitafloxacin against atypical bacteria (2009-2014) and comparison between susceptibility of clinical isolates in 2009 and 2012. Jpn. J. Antibiot. 69, 131-142.

Asakura, T., Suzuki, S., Fukano, H., Okamori, S., Kusumoto, T., Uwamino, Y., et al. (2019). Sitafloxacin-containing regimen for the treatment of refractory 
Mycobacterium avium complex lung disease. Open Forum Infect. Dis. 6:ofz108. doi: 10.1093/ofid/ofz108

Choi, G.-E., Min, K.-N., Won, C.-J., Jeon, K., Shin, S. J., and Koh, W.-J. (2012a). Activities of moxifloxacin in combination with macrolides against clinical isolates of Mycobacterium abscessus and Mycobacterium massiliense. Antimicrob. Agents Chemother. 56, 3549-3555. doi: 10.1128/AAC.00685-12

Choi, G.-E., Shin, S. J., Won, C.-J., Min, K.-N., Oh, T., Hahn, M.-Y., et al. (2012b). Macrolide treatment for Mycobacterium abscessus and Mycobacterium massiliense infection and inducible resistance. Am. J. Respir. Crit. Care Med. 186, 917-925. doi: 10.1164/rccm.201111-2005OC

Cowman, S., Van Ingen, J., Griffith, D. E., and Loebinger, M. R. (2019). Nontuberculous mycobacterial pulmonary disease. Eur. Respir. J. 54:1900250. doi: 10.1183/13993003.00250-2019

Daley, C. L., Iaccarino, J. M., Lange, C., Cambau, E., Wallace, R. J., Andrejak, C., et al. (2020). Treatment of nontuberculous mycobacterial pulmonary disease: an official ATS/ERS/ESCMID/IDSA clinical practice guideline. Clinical Infect. Dis. 71, 905-913. doi: 10.1093/cid/ciaa1125

Degiacomi, G., Sammartino, J. C., Chiarelli, L. R., Riabova, O., Makarov, V., and Pasca, M. R. (2019). Mycobacterium abscessus, an emerging and worrisome pathogen among cystic fibrosis patients. Int. J. Mol. Sci. 20:5868. doi: 10.3390/ ijms20235868

Fletcher, L. A., Chen, Y., Whitaker, P., Denton, M., Peckham, D. G., and Clifton, I. J. (2016). Survival of Mycobacterium abscessus isolated from people with cystic fibrosis in artificially generated aerosols. Eur. Respir. J. 48, 1789-1791. doi: 10.1183/13993003.00849-2016

Guo, Q., Chen, J., Zhang, S., Zou, Y., Zhang, Y., Huang, D., et al. (2020). Efflux pumps contribute to intrinsic clarithromycin resistance in clinical. Mycobacterium abscessus isolates. Infect. Drug Resist. 13, 447-454. doi: 10.2147/ IDR.S239850

Haworth, C. S., Banks, J., Capstick, T., Fisher, A. J., Gorsuch, T., Laurenson, I. F., et al. (2017). British thoracic society guidelines for the management of nontuberculous mycobacterial pulmonary disease (NTM-PD). Thorax 72, ii1-ii64. doi: 10.1136/thoraxjnl-2017-210927

Johansen, M. D., Herrmann, J.-L., and Kremer, L. (2020). Non-tuberculous mycobacteria and the rise of Mycobacterium abscessus. Nat.Rev. Microbiol. 18, 392-407. doi: 10.1038/s41579-020-0331-1

Kamada, K., Yoshida, A., Iguchi, S., Arai, Y., Uzawa, Y., Konno, S., et al. (2021). Nationwide surveillance of antimicrobial susceptibility of 509 rapidly growing mycobacteria strains isolated from clinical specimens in Japan. Sci. Rep. 11:12208. doi: 10.1038/s41598-021-91757-4

Kaushik, A., Ammerman, N. C., Tasneen, R., Story-Roller, E., Dooley, K. E., Dorman, S. E., et al. (2017). In vitro and in vivo activity of biapenem against drug-susceptible and rifampicin-resistant Mycobacterium tuberculosis. J. Antimicrob. Chemother. 72, 2320-2325. doi: 10.1093/jac/dkx152

Koh, W.-J., Jeon, K., Lee, N. Y., Kim, B.-J., Kook, Y.-H., Lee, S.-H., et al. (2011). Clinical significance of differentiation of Mycobacterium massiliense from Mycobacterium abscessus. Am. J. Respir. Crit. Care Med. 183, 405-410. doi: 10.1164/rccm.201003-0395OC

Li, Y., Yin, Y., Peng, X., Zheng, H., Fu, F., Liu, Z., et al. (2021a). A randomized, active-controlled, multicentre clinical trial to evaluate the efficacy and safety of oral sitafloxacin versus levofloxacin in Chinese adults with acute uncomplicated or complicated urinary tract infection. Ann. Med. 53, 217-226. doi: 10.1080/ 07853890.2020 .1861322

Li, Y., Zhu, D., Peng, Y., Tong, Z., Ma, Z., Xu, J., et al. (2021b). A randomized, controlled, multicenter clinical trial to evaluate the efficacy and safety of oral sitafloxacin versus moxifloxacin in adult patients with community-acquired pneumonia. Curr. Med. Res. Opin. 37, 693-701. doi: 10.1080/03007995.2021. 1885362

Miyazaki, T., Nakamura, S., Hashiguchi, K., Kobayashi, T., Fukushima, K., Fukuda, Y., et al. (2019). The efficacy and safety of sitafloxacin and garenoxacin for the treatment of pneumonia in elderly patients: a randomized, multicenter, open-label trial. J. Infect. Chemother. 25, 886-893. doi: 10.1016/j.jiac.2019.04.019
Mori, H., Suzuki, H., Matsuzaki, J., Masaoka, T., and Kanai, T. (2020). 10-year trends in Helicobacter pylori eradication rates by sitafloxacin-based third-line rescue therapy. Digestion 101, 644-650. doi: 10.1159/000501610

Mourad, A., Baker, A. W., and Stout, J. E. (2021). Reduction in expected survival associated with nontuberculous mycobacterial pulmonary disease. Clinical Infect. Dis. 72, e552-e557. doi: 10.1093/cid/ciaa1267

Nakajima, T., Okui, T., Ito, H., Nakajima, M., Honda, T., Shimada, Y., et al. (2016). Microbiological and clinical effects of sitafloxacin and azithromycin in periodontitis patients receiving supportive periodontal therapy. Antimicrob. Agents Chemother. 60, 1779-1787. doi: 10.1128/AAC.02575-15

Oka, K., Morioka, H., Eguchi, M., Sato, Y., Tetsuka, N., Iguchi, M., et al. (2021). A case report of bursitis, bacteremia, and disseminated infection of Mycobacteroides (Mycobacterium) abscessus subsp. massiliense. Int. Med. 60, 6189-6220. doi: 10.2169/internalmedicine.6189-20

Saito, H., Tomioka, H., Sato, K., and Dekio, S. (1994). In vitro and in vivo antimycobacterial activities of a new quinolone, DU-6859a. Antimicrob. Agents Chemother. 38, 2877-2882. doi: 10.1128/AAC.38.12.2877

Sato, K., Tomioka, H., Sano, C., Shimizu, T., Sano, K., Ogasawara, K., et al. (2003). Comparative antimicrobial activities of gatifloxacin, sitafloxacin and levofloxacin against Mycobacterium tuberculosis replicating within Mono Mac 6 human macrophage and A-549 type II alveolar cell lines. J. Antimicrob. Chemother. 52, 199-203. doi: 10.1093/jac/dkg343

Stout, J. E., Koh, W.-J., and Yew, W. W. (2016). Update on pulmonary disease due to non-tuberculous mycobacteria. Int. J. Infect. Dis. 45, 123-134. doi: 10.1016/j. ijid.2016.03.006

Takano, K., Shimada, D., Kashiwagura, S., Kamioka, Y., Hariu, M., Watanabe, Y., et al. (2021). Severe pulmonary Mycobacterium abscessus cases due to co-infection with other microorganisms well treated by clarithromycin and stafloxacin in Japan. Int. Med. Case Rep. J. 14, 465-470. doi: 10.2147/IMCRJ. S321969

Tomioka, H., Sato, K., Akaki, T., Kajitani, H., Kawahara, S., and Sakatani, M. (1999). Comparative in vitro antimicrobial activities of the newly synthesized quinolone HSR-903, sitafloxacin (DU-6859a), gatifloxacin (AM-1155), and levofloxacin against Mycobacterium tuberculosis and Mycobacterium avium complex. Antimicrob. Agents Chemother. 43, 3001-3004. doi: 10.1128/AAC.43. 12.3001

Woods, G. L., Brown-Elliott, B. A., Conville, P. S., Desmond, E. P., Hall, G. S., Lin, G., et al. (2011). Susceptibility Testing of Mycobacteria, Nocardiae, and Other Aerobic Actinomycetes. Wayne, PA: Clinical and Laboratory Standards Institute.

Wu, G., Wu, L., Hu, X., Zhou, H., Liu, J., Zhu, M., et al. (2014). Pharmacokinetics and safety of sitafloxacin after single oral doses in healthy volunteers. Int. J. Clin. Pharmacol. Ther. 52, 1037-1044. doi: 10.5414/CP202147

Zhang, S., Zou, Y., Guo, Q., Chen, J., Xu, L., Wan, X., et al. (2020). AR-12 exhibits direct and host-targeted antibacterial activity toward Mycobacterium abscessus. Antimicrob. Agents Chemother. 64, e236-e320. doi: 10.1128/AAC.00236-20

Conflict of Interest: The authors declare that the research was conducted in the absence of any commercial or financial relationships that could be construed as a potential conflict of interest.

Publisher's Note: All claims expressed in this article are solely those of the authors and do not necessarily represent those of their affiliated organizations, or those of the publisher, the editors and the reviewers. Any product that may be evaluated in this article, or claim that may be made by its manufacturer, is not guaranteed or endorsed by the publisher.

Copyright () $2022 \mathrm{He}$, Guo, Zhao, Xu, Fan, Wu, Zhang, Li and Chu. This is an open-access article distributed under the terms of the Creative Commons Attribution License (CC BY). The use, distribution or reproduction in other forums is permitted, provided the original author(s) and the copyright owner(s) are credited and that the original publication in this journal is cited, in accordance with accepted academic practice. No use, distribution or reproduction is permitted which does not comply with these terms. 